\title{
EUS-guided hepaticogastrostomy for hepatic hilar obstruction
}

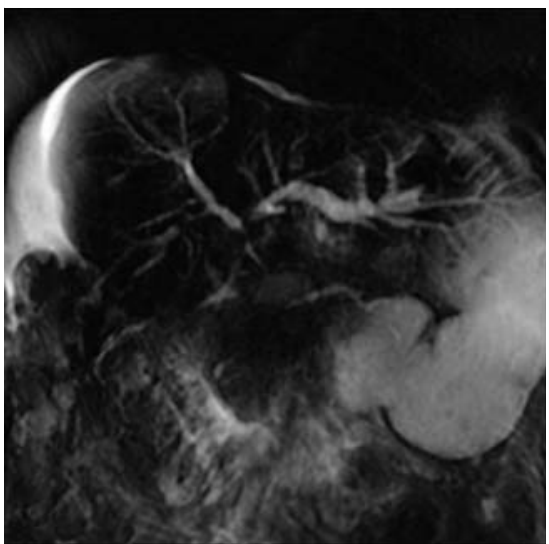

Fig. 1 Magnetic resonance cholangiopancreatography demonstrated bile duct stenosis from the common bile duct to the left and right hepatic bile ducts.

EUS-guided hepaticogastrostomy (EUSHGS) was recently developed to treat biliary decompression in patients with biliary obstruction, as an alternative to (failed) endoscopic biliary drainage or percutaneous transhepatic biliary drainage (PTBD), or when the papilla is inaccessible [1]. In addition, EUS-HGS has the advantage of internal drainage compared with PTBD. However, because EUS-HGS is a method of left intrahepatic biliary drainage, it is not usually indicated in patients with hepatic hilar obstruction [2]. We describe a case of successfully biliary drainage for hepatic hilar obstruction using a novel uncovered metallic stent with a fine-gauge delivery system.

A 57-year-old woman was admitted to our institution with obstructive jaundice that developed during chemotherapy for colon cancer. CT showed a mass at the hepatic hilum with multiple metastatic liver tumors. Magnetic resonance cholangiopancreatography demonstrated bile duct stenosis from the common bile duct to the left and right hepatic bile ducts, caused by hilar metastasis from the colon cancer ( Fig.1). Because endoscopic biliary drainage failed, we decided to perform EUS-HGS. First, the intrahepatic bile duct (segment 3: B3) was punctured using a 19G (Sono Tip Pro Control 19G; MediGlobe $\mathrm{GmbH}$, Rosenheim, Germany) needle, using Doppler ultrasonography to avoid any intervening vessels ( $\bullet$ Fig. 2 a). Bile juice was aspirated, and a small amount of contrast medium was injected

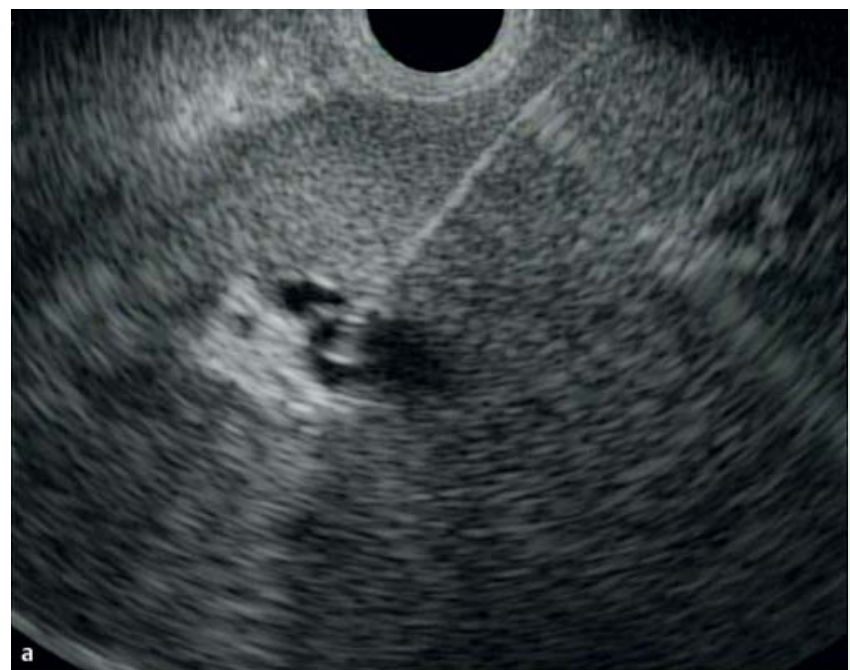

Fig. 2 a The intrahepatic bile duct (segment 3) was punctured using a $19 \mathrm{G}$ fine-needle aspiration needle. b After aspiration of bile juice, a small amount of contrast medium was injected.

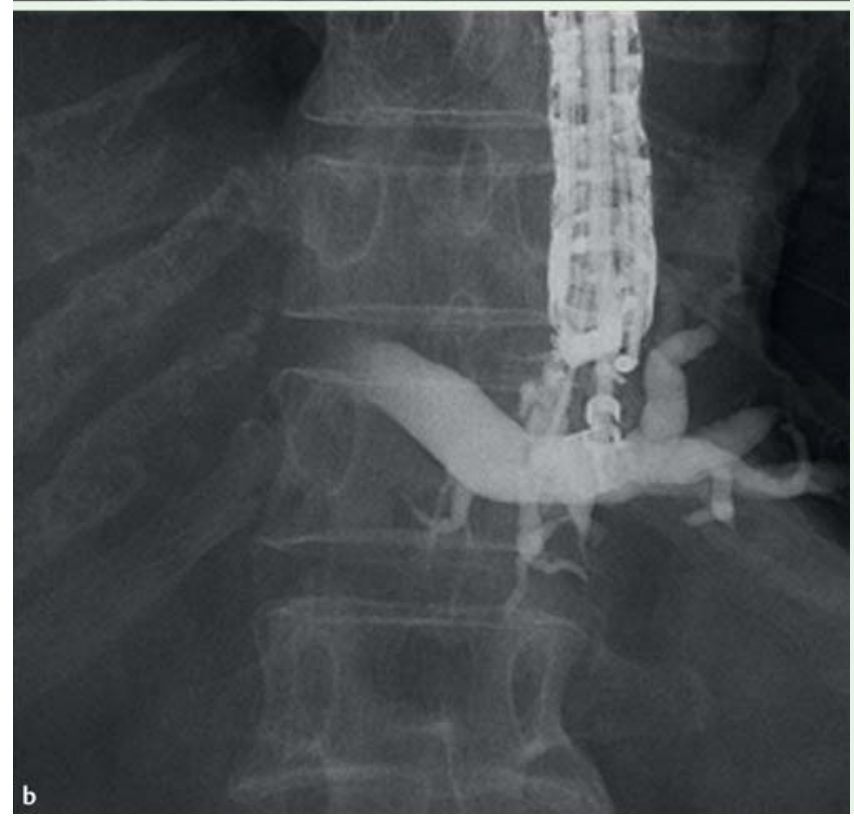

( $\bullet$ Fig. 2 b). A 0.025-inch guidewire (VisiGlide, angle type; Olympus Medical Systems, Tokyo, Japan) was placed into the right intrahepatic bile duct ( $\bullet$ Fig.3a), and then we inserted the delivery system of the uncovered metallic stent $(8 \mathrm{~mm} \times 8 \mathrm{~cm}$, Zilver 635 biliary self-expanding stent; Cook Medical, Bloomington, IN, USA). Because this delivery system is only $6 \mathrm{Fr}$ in diameter, and it is a stiff delivery system, it was not necessary to dilate the fistula for its insertion, and we were able to advance to the right hepatic bile duct ( Fig.3b). Finally, we performed EUS-HGS using a fully covered, self-expandable metallic stent (end bare type, $10 \mathrm{~mm} \times 10 \mathrm{~cm}$, Niti-S biliary covered stent; TaeWoong Medical, Seoul, Korea) with the same guidewire as described ( $\bullet$ Fig.3c) [3]. This second stent was placed to course from the left hepatic bile duct to the stomach ( $\bullet$ Video 1 ).

\section{Video 1}

The guidewire was advanced into the right hepatic bile duct and contrast medium injected into the bile ducts. A stenosis was seen extending from the common bile duct into the right and left bile ducts. A fine-gauge delivery system was inserted across the hepatic hilum, and an uncovered metallic stent was deployed. Finally, endoscopic ultrasound-guided hepaticogastrostomy was performed using a fully covered metallic stent. 


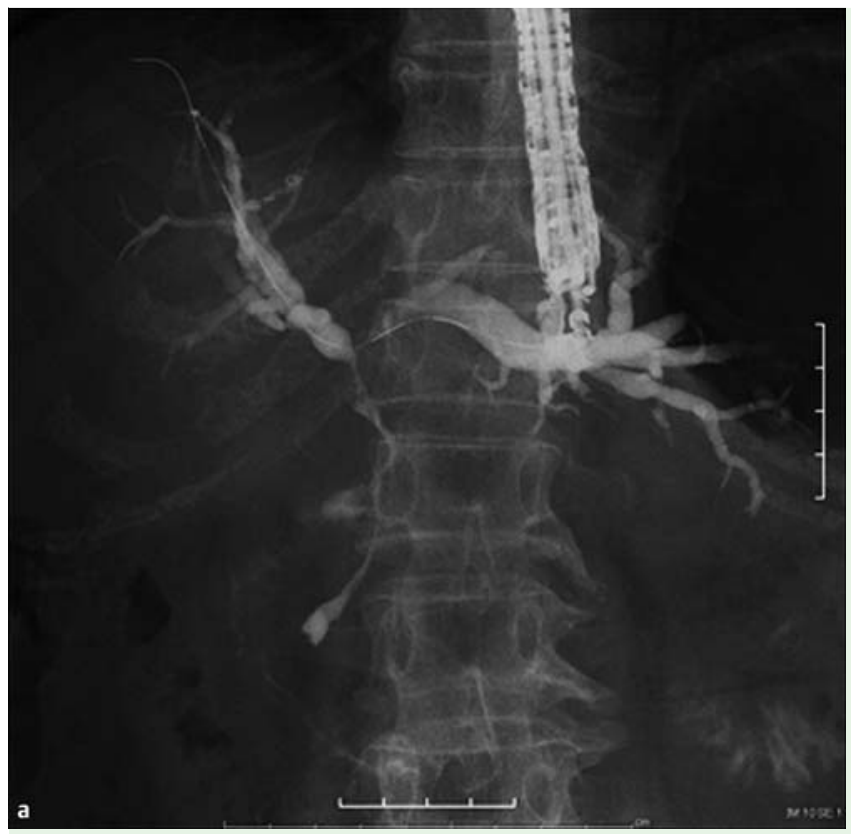

Fig. 3 a A guidewire was advanced from the left intrahepatic bile duct to the right intrahepatic bile duct. b The fine-gauge (6-Fr) delivery system was successfully inserted. c Finally, endoscopic ultrasound-guided hepaticogastrostomy was performed.
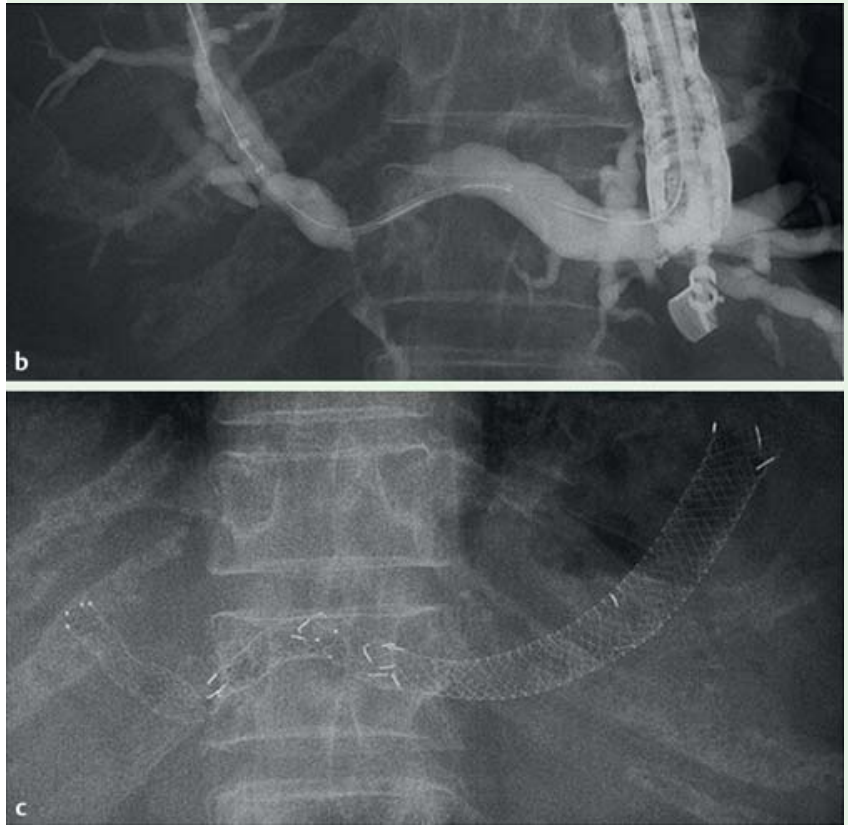

EUS-HGS is an effective alternative drainage method. For obstructive jaundice caused by hepatic hilar obstruction, EUSHGS using this fine-gauge delivery system might be indicated, as in the case presented here.

Endoscopy_UCTN_Code_TTT_1AS_2AD

Competing interests: None

\section{Takeshi Ogura, Daisuke Masuda, Akira Imoto, Eiji Umegaki, Kazuhide Higuchi}

Second Department of Internal Medicine, Osaka Medical College, Osaka, Japan

\section{References}

1 Giovannini M, Dotti M, Boris E et al. Hepaticogastrostomy by echoendoscopy as a palliative treatment in a patient with metastatic biliary obstruction. Endoscopy 2003; 35: $1076-1078$

2 Giovannini M, Bories E. EUS-guided biliary drainage. Gastroenterol Res Pract 2012; 104: 348719

3 Itoi T, Isayama H, Sofuni A et al. Stent selection and tips on placement technique of EUS-guided biliary drainage: transduodenal and transgastric stenting. J Hepatobiliary Pancreatic Sci 2011; 18: 664-672

\section{Bibliography}

DOI http://dx.doi.org/

10.1055/s-0033-1359133

Endoscopy 2014; 46: E32-E33

(c) Georg Thieme Verlag KG

Stuttgart · New York

ISSN 0013-726X

\section{Corresponding author \\ Takeshi Ogura, MD, PhD}

Second Department of Internal Medicine

Osaka Medical College

2-7 Daigaku-machi, Takatsuki-shi

Osaka 569-8686

Japan

Fax: +81-7-26846532

oguratakeshi0411@yahoo.co.jp 